\title{
Duplicação total do fêmur. Relato de caso com 23 anos de seguimento*
}

\section{Total Duplication of the Femur. Case Report with 23 Years of Follow-up}

\author{
Rafael Garcia de Oliveira ${ }^{1}$ Giampaulo Marcelo Catelan ${ }^{1}$ Cicero Ricardo Gomes ${ }^{1}$ (이 \\ ${ }^{1}$ Departamento de Ortopedia, Rede Sarah de Hospitais de \\ Reabilitação, Brasília, Distrito Federal, Brasil \\ Rev Bras Ortop \\ Endereço para correspondência Rafael Garcia de Oliveira, MD, \\ Departamento de Ortopedia, Rede Sarah de Hospitais de Reabilitação, \\ SMHS 501, Bloco A-, Brasília, Distrito Federal, 70.335-901, Brasil \\ (e-mail: rafagaroli@gmail.com).
}

\section{Resumo \\ Palavras-chave \\ - fêmur \\ - dismetria \\ - escoliose \\ - deformidades congênitas dos membros}

A duplicação do fêmur é raríssima, e os poucos casos descritos são de duplicações parcial, proximal ou distal. Segundo a nossa revisão de literatura, esse é o primeiro relato de duplicação total. O paciente foi acompanhado, sem tratamento cirúrgico, por 23 anos. Na última visita, queixava-se de dor lombar marcada discrepância dos membros inferiores. A avaliação clínico-radiográfica mostrava um joelho direito com dois côndilos femorais separados que se articulavam com a tíbia proximal e uma patela hipoplásica, e limitação da flexão do joelho em $95^{\circ}$. O paciente apresentava dismetria dos membros inferiores medida em $17 \mathrm{~cm}$, o que resultava em obliquidade pélvica e escoliose dextroconvexa. O paciente recebeu uma ortoprótese de $16 \mathrm{~cm}$ que trouxe melhora do padrão de marcha e resolução da queixa de lombalgia.

Femur duplication is very rare, and the few cases described are of partial, proximal or distal duplications. Based on our literature review, the present is the first report of a case of total duplication. The patient was followed up, without surgical treatment, for 23 years. At the last visit, the patient complained of low-back pain and marked discrepancy in the lower limbs. A clinic-radiographic evaluation showed a right knee with two separate femoral condyles that articulated with the proximal tibia, a hyporadioactive patella, and a limitation in knee flexion at $95^{\circ}$. The patient presented lower-limb dysmetria, measuring $17 \mathrm{~cm}$, resulting in pelvic obliquity and dextroconvex scoliosis. The patient received a $16 \mathrm{~cm}$ orthoprosthesis that improved the gait pattern and resolved the complaint of low-back pain.
Trabalho desenvolvido no Departamento de Ortopedia, Rede Sarah de Hospitais de Reabilitação, Brasília, Distrito Federal, Brasil. recebido

21 de Dezembro de 2020

aceito

14 de Maio de 2021
DOI https://doi.org/

10.1055/s-0041-1735139. ISSN 0102-3616. (c) 2021. Sociedade Brasileira de Ortopedia e Traumatologia. All rights reserved.

This is an open access article published by Thieme under the terms of the Creative Commons Attribution-NonDerivative-NonCommercial-License, permitting copying and reproduction so long as the original work is given appropriate credit. Contents may not be used for commercial purposes, or adapted, remixed, transformed or built upon. (https://creativecommons.org/ licenses/by-nc-nd/4.0/)

Thieme Revinter Publicações Ltda., Rua do Matoso 170, Rio de Janeiro, RJ, CEP 20270-135, Brazil 


\section{Introdução}

A deformidade femoral mais comum é o fêmur curto congênito. Alguns autores descreveram raros casos de bifurcação femoral distal, que é característica do complexo de GollopWolfgang, uma síndrome genética rara, geralmente associada a deformidades no membro ipsilateral, tais como aplasia tibial, hipoplasia, ou pé equinovaro. ${ }^{1-4}$ Houve ainda um caso de duplicação femoral proximal relatado em 2009; no entanto, duplicações femorais totais nunca foram descritas na literatura.

\section{Relato de Caso}

Um paciente foi avaliado no setor de ortopedia da Rede Sarah de Hospitais de Reabilitação, em Brasília, aos 35 dias de vida por uma aparência estética anormal e encurtamento do membro inferior direito. Tratava-se do segundo filho de uma mãe saudável, fruto de gravidez sem intercorrências, nascido a termo, e sem fatores de risco identificáveis para anomalias congênitas. Não havia parentes conhecidos com deformidade nos membros ou histórico genético relevante. 0 membro direito era $3 \mathrm{~cm}$ mais curto e levemente mais fino, sendo a redução do comprimento concentrada no fêmur, ao passo que as tíbias e pés eram simétricos e sem deformidades ( - Figura 1). Quadris e joelhos apresentavam amplitude de movimento regular e simétrica ao lado oposto. Não houve achados nos sistemas renal, cardiovascular, gastrointestinal e nervoso, e não foram observadas malformações nos membros superiores. O menino apresentou um desenvolvimento neuropsicomotor global normal, e começou a andar aos 14 meses.

Com 1 ano de idade, uma radiografia mostrou duplicação total do fêmur. Na região proximal, o fêmur medial se articulava no quadril sem anormalidades, apesar de certo valgo do colo femoral ( - Figura 2), e o fêmur lateral estava no nível do trocanter maior. A epífise proximal da tíbia direita era hipoplásica e inclinada na direção do fêmur medial. A fíbula não mostrava deformidades evidentes. Uma avaliação radiográfica da coluna vertebral não mostrou desvios ou anomalias congênitas.



Fig. 1 Paciente com 35 dias de vida, apresentando discrepância entre os membros, sendo o direito mais curto.

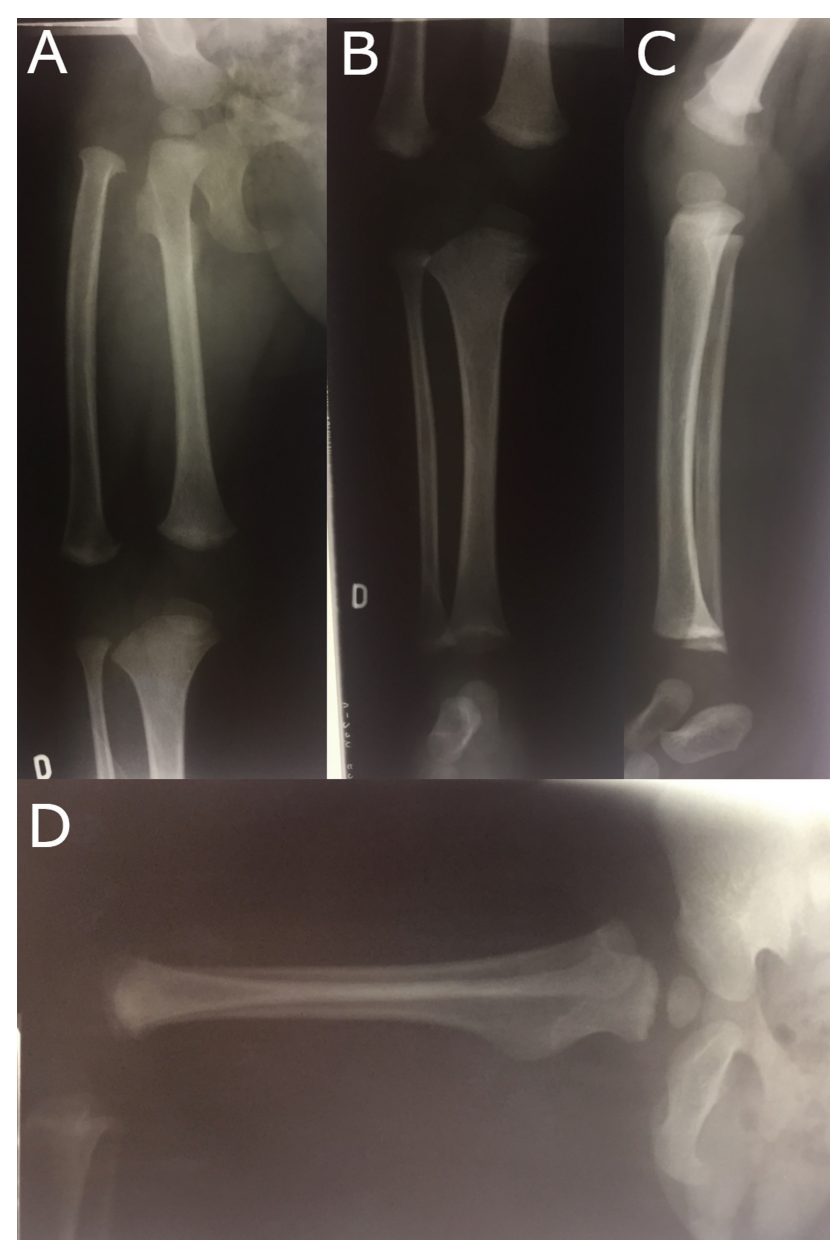

Fig. 2 Radiografia com 1 ano de idade. (A) Quadril direito e fêmur direito em incidência anteroposterior (AP). (B) Fêmur distal e tíbia em incidência AP. (C) Fêmur distal e tíbia em perfil. (D) Quadril direito e fêmur em perfil.

O próximo encontro ocorreu aos 3 anos de idade; naquele momento, a disparidade nos membros inferiores havia aumentado para $6 \mathrm{~cm}$ (-Figura 3). Observou-se

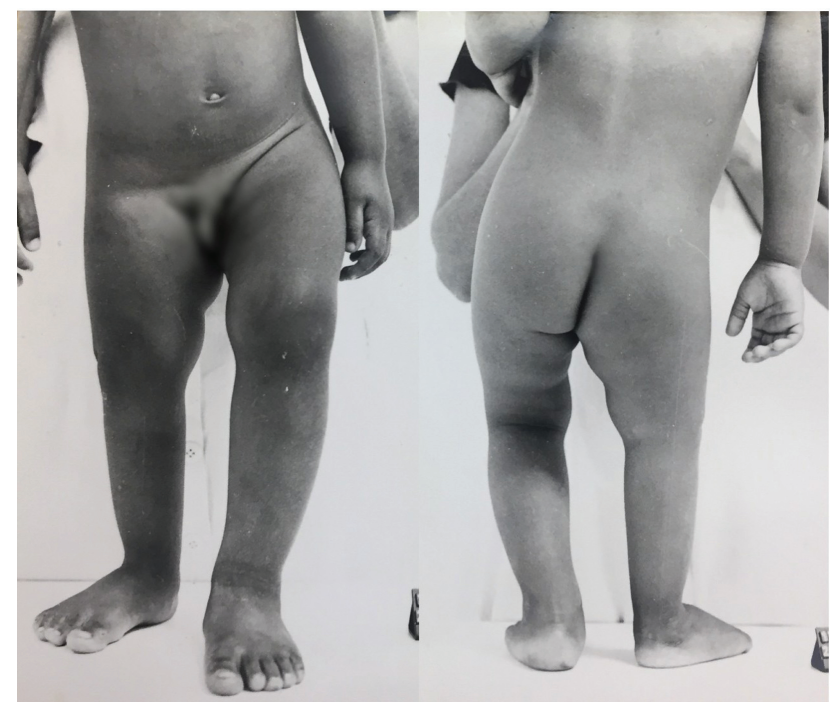

Fig. 3 Imagens clínicas do paciente com 3 anos de idade, mostrando uma discrepância de $6 \mathrm{~cm}$ no comprimento das pernas. 


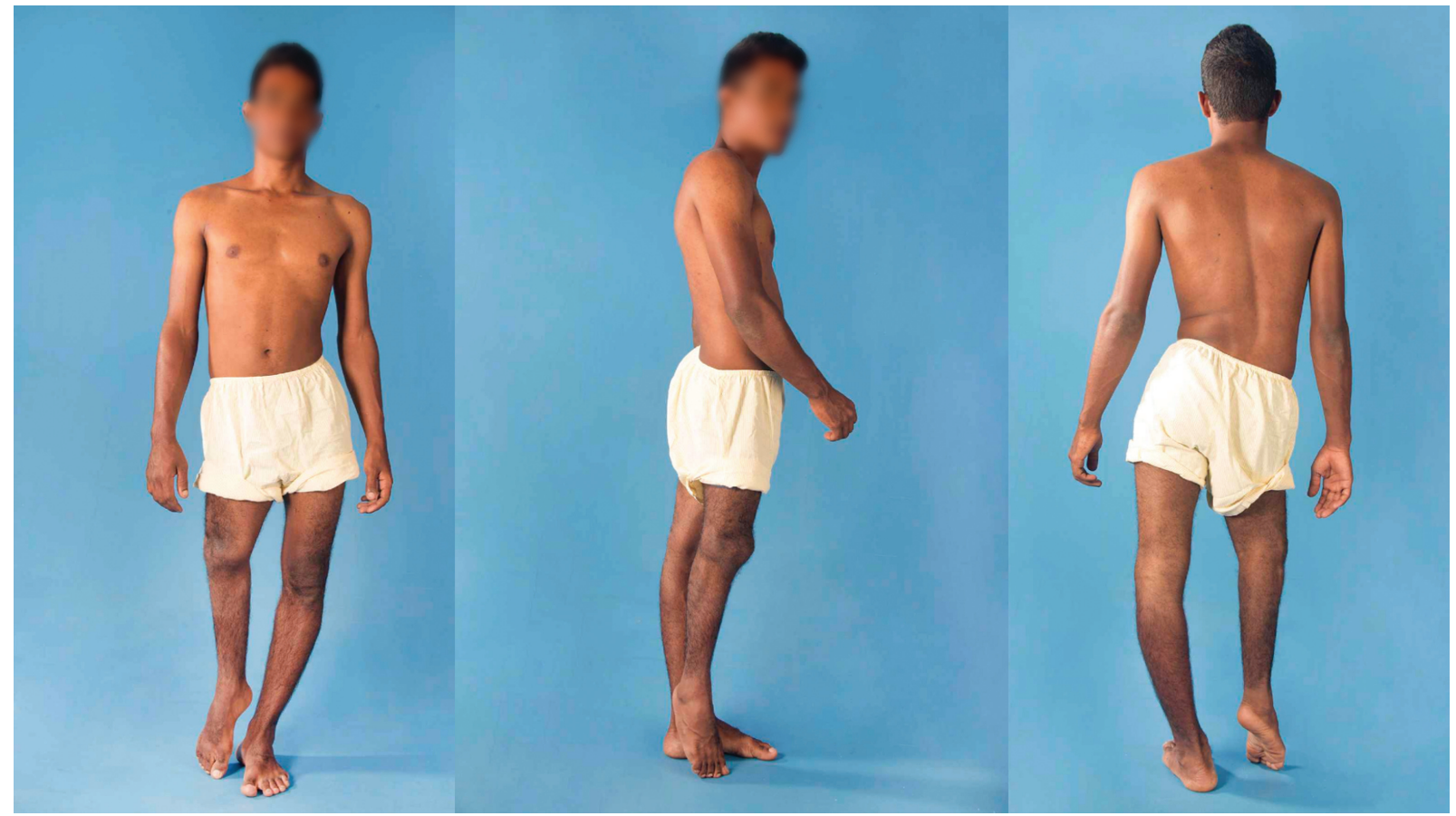

Fig. 4 Imagens clínicas do paciente com 23 anos, mostrando uma discrepância de $17 \mathrm{~cm}$ no comprimento das pernas.

perda na amplitude de flexão do joelho no lado direito, que estava entre $0^{\circ}$ e $70^{\circ}$. Enquanto caminhava, o paciente mantinha o pé direito na posição de equino (redutível), e o joelho esquerdo, semiflexionado para compensar a discrepância.

Radiografias mostraram a presença de epífise distal no fêmur medial, e a epífise proximal da tíbia manteve seu desenvolvimento concentrado no lado medial. Usando o método multiplicador de Paley para a previsão de comprimento aos 3 anos de idade, a discrepância esperada seria de $13,4 \mathrm{~cm}$ na maturidade esquelética. 0 paciente se adaptou bem a uma compensação de sapato de $5 \mathrm{~cm}$. Após essa visita, o paciente não retornou pelos próximos vinte anos.

Em agosto de 2016, aos 23 anos, o paciente buscou novo atendimento. Sua principal preocupação era a discrepância dos membros inferiores e dor lombar esporádica. Ele não usava nenhuma compensação, sendo observada rotação externa do membro às custas do quadril/fêmur, e, durante a marcha, mantinha o joelho esquerdo semi-flexionado e o pé direito em posição equina. Além disso, havia evidente escoliose com convexidade à direita e obliquidade pélvica (-Figura 4). À direita, a amplitude de flexo-extensão do joelho era de $0^{\circ}$ a $95^{\circ}$, e a do quadril, de $-20^{\circ}$ a $45^{\circ}$, comparada a $-30^{\circ}$ a $90^{\circ}$ no lado esquerdo.

Uma radiografia mostrou um colo femoral valgo à direita, com articulação normal do quadril, um joelho com dois côndilos femorais separados que se articulavam com a tíbia proximal, e uma patela hipoplásica (-Figura 5). O domo do tálus apresentava configuração esférica, articulando-se com uma superfície côncava na tíbia, configurando tornozelo esferoide (tipo ball and socket). As medidas radiográficas em ortostatismo foram $67 \mathrm{~cm}$ (fêmur: $33 \mathrm{~cm}$; tíbia: $34 \mathrm{~cm}$ ) à direita, e $84 \mathrm{~cm}$ (fêmur: $46 \mathrm{~cm}$; tíbia: $38 \mathrm{~cm}$ ) à esquerda, revelando discrepância de $17 \mathrm{~cm}$, semelhante à medida clínica.

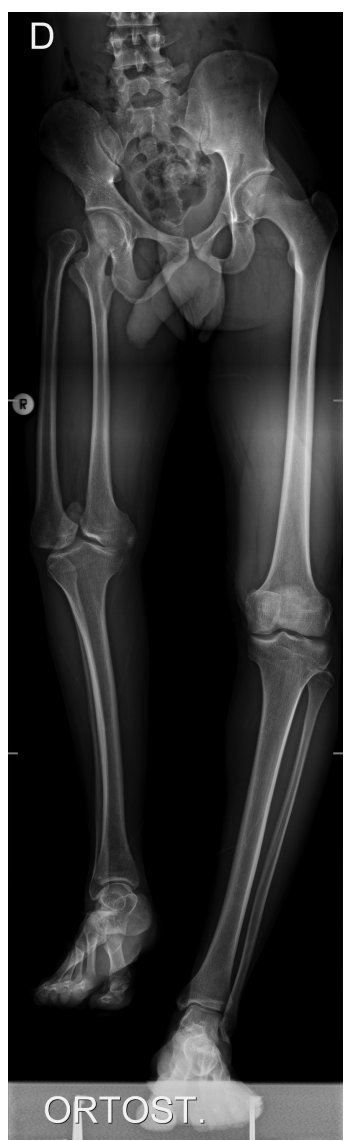

Fig. 5 Radiografia em AP dos membros inferiores, aos 23 anos de idade. A imagem mostra o colo femoral valgo à direita, com articulação normal do quadril, um joelho com dois côndilos femorais separados se articulando com a tíbia proximal, e uma patela hipoplásica. O domo do tálus tem aspecto esférico, e articula-se com uma superfície côncava na tíbia, configurando tornozelo esferoide (tipo ball and socket). A medida do membro inferior direito é de $67 \mathrm{~cm}$ (fêmur: 33 cm; tíbia 34: cm), e, do esquerdo, de 84 cm (fêmur: 46 cm; tíbia: $38 \mathrm{~cm}$ ). 
Uma ressonância magnética (RM) mostrou côndilos femorais com superfície irregular, e cartilagem fina nas porções posterolateral do côndilo lateral e anteromedial do côndilo medial. Existem contornos de menisco, que predominam nas porções anterior dos compartimentos lateral e posterior do compartimento medial. Uma estrutura de sinal baixo é identificada em topografia intra-articular com trajeto oblíquo, inserido-se na face medial do côndilo femoral lateral e no platô tibial posterior.

Após receber uma ortoprótese de $16 \mathrm{~cm}$, houve resolução da queixa de lombalgia.

\section{Discussão}

Este é o primeiro relato da literatura de uma duplicação total do fêmur levando à formação de um joelho peculiar. Nas imagens, pode-se observar a evolução do quadro, desde os primeiros dias após o nascimento até a idade adulta. Apesar das repercussões clínicas da grande dismetria, o paciente apresentou um bom desenvolvimento muscular global, e trabalha como auxiliar de pedreiro, função com exigência física elevada. Após o uso da ortoprótese por três meses, o paciente referiu melhora completa do quadro de lombalgia, apresentando-se completamente assintomático nesse momento. Por conta da estrutura anatômica do joelho, os autores não consideraram qualquer abordagem cirúrgica, pois um eventual alongamento ósseo poderia resultar em piora funcional significativa. $\mathrm{O}$ tratamento com ortoprótese mostrou-se eficaz em restabelecer a qualidade de vida do paciente.

Foram feitos poucos relatos de bifurcação distal do fêmur desde a primeira descrição de Erlich em 1889. De maneira geral, ela ocorre associada a outras deformidades no membro ipsilateral, como aplasia tibial, hipoplasia do pé, e pé equinovaro, e é característica do complexo de Gollop-Wolfgang. ${ }^{1-4}$ Podem também estar associada a malformações dos membros superiores. ${ }^{5}$

Há algumas teorias sobre a etiologia da bifurcação femoral isolada, e é provável que seja heterogênea, como postulado por Bodurtha et al. ${ }^{6}$ Neuropatia embriológica pode levar a uma ampla variedade de anormalidades, dependendo dos nervos envolvidos e de sua respectiva inervação. $O$ crescimento de um órgão ou parte dele está relacionado ao seu suprimento nervoso, devido à sua função de coestimulador do crescimento.

Ogden $^{3}$ relatou um caso de bifurcação femoral e hemimelia tibial ipsilateral. Ele propôs que a bifurcação femoral resultava da divisão do mesoderma do botão embrionário que origina o membro, resultando em um desenvolvimento independente de cada uma dessas metades. Essa divisão pode ser produzida mecanicamente a partir da pressão de bandas amnióticas, levando à duplicação total ou parcial do membro.

Osuji et al. ${ }^{7}$ relataram o caso de um paciente que apresentava duplicação proximal do fêmur, e que foi submetido a excisão de uma das cabeças e colo do fêmur associada a uma osteotomia de derrotação, osteotomia pélvica tipo Salter, e tenotomia de adutores devido a anteversão remanescente da cabeça femoral de $70^{\circ}$. Comparado ao caso atual, a excisão completa do fêmur não era uma opção, pois a porção lateral formava o côndilo lateral do fêmur, e a porção medial, a cabeça e o colo do fêmur.

Como esse paciente não foi submetido a intervenção cirúrgica, é possível observar a história natural dessa malformação incomum ao longo de 23 anos.

\section{Declarações Éticas}

O presente estudo foi aprovado pelo Comitê de Ética da Associação das Pioneiras Sociais (número do processo CAAE: 34092620.0.0000.0022).

\section{Suporte Financeiro}

Não houve suporte financeiro de fontes públicas, comerciais, ou sem fins lucrativos.

\section{Conflito de Interesses}

Os autores declaram não haver conflito de interesses.

\section{Referências}

1 Wolfgang GL. Complex congenital anomalies of the lower extremities: femoral bifurcation, tibial hemimelia, and diastasis of the ankle. Case report and review of the literature. J Bone Joint Surg Am 1984;66(03):453-458

2 Kotakemori K, Ito J. Femoral bifurcation with tibial aplasia. A case report and review of the literatures. Clin Orthop Relat Res 1978; (135):26-28

3 Ogden JA. Ipsilateral femoral bifurcation and tibial hemimelia. A case report. J Bone Joint Surg Am 1976;58(05):712-713

4 Dhanakodi N, Tripathy SK. Gollop-Wolfgang syndrome. Indian J Med Res 2014;139(06):963-964

5 Gollop TR, Lucchesi E, Martins RMM, Nione AS. Familial occurrence of bifid femur and monodactylous ectrodactyly. Am J Med Genet 1980;7(03):319-322

6 Bodurtha J, Coutinho M, Benator R, Nogi J, Sholley M, Nance WE. Femoral duplication: a case report. Am J Med Genet 1989;33(02): 165-169

7 Osuji CK, Chafik D, Moseley CF. Unilateral proximal bifurcation of the femur. J Pediatr Orthop 2009;29(07):695-697 